\title{
Cross Sectional Data on Profile of Coronary Artery Disease in Human Immunodeficiency Virus Infection Patients in a Tertiary Care Hospital of Central India
}

\author{
Atul Singh Rajput ${ }^{1}$, Sunil Washimkar ${ }^{2}$, Gunjan S Dalal ${ }^{3}$, Pradeep Deshmukh ${ }^{4}$ \\ ${ }^{1}$ Assistant Professor, Dept of Cardiology, GMC SSH Nagpur, India \\ ${ }^{2}$ Associate Professor, Dept of Cardiology, GMC SSH Nagpur, India \\ ${ }^{3}$ Assistant Professor, Dept of Medicine, IGGMC Nagpur, India \\ ${ }^{4}$ Professor and Head, Dept of Cardiology, GMC \& SSH, Nagpur
}

Corresponding Author: Sunil Washimkar

\begin{abstract}
Background: Human immunodeficiency virus infection leads to a variety of cardiovascular manifestations. Pericardial effusion, systemic hypertension, dilated cardiomyopathy, coronary artery disease, pulmonary hypertension, primary as well as secondary cardiac tumours etc are seen in about $30 \%$ of patients and the incidence has increased with improved longevity. Coronary artery disease ranging from subclinical atherosclerosis to coronary plaque rupture causing acute coronary syndrome have been documented. The main underlying pathophysiological process is a state of perennial inflammation which leads to initiation and acceleration of coronary atherosclerosis.

Objective: To study the coronary angiographic profile in HIV patients.

Methodology: HIV patients undergoing coronary angiography were assessed clinically, biochemically and finally by coronary angiography to see the coronary pathoanatomy.

Results: Of the 37 HIV patients studied between Jan to Dec 2019, the mean age was 47 years.(19-66 years). Two thirds were males; most common traditional risk factor was dyslipidemia followed by hypertension. Most patients were already on ART and the CD 4 counts ranged from 67-366cells/cumm. Anterior wall MI and unstable angina were the most common clinical presentations. Coronary angiography revealed diffuse disease (both insignificant and significant) of the left anterior
\end{abstract}

descending artery as the most common pattern of angiographic involvement.

Conclusion: Coronary artery disease both subclinical and syndromic occurs with increased frequency in HIV patients. The dominant angiographic presentation correlates with the clinical presentation and in our study, left anterior descending artery was more frequently diseased as compared to other coronaries. The most common coronary angiographic pattern was the presence of ectasia and diffuse disease.

Keywords: Human immunodeficiency virus, cardiovascular manifestations, coronary artery disease, coronary angiography.

\section{INTRODUCTION}

Human immunodeficiency virus (HIV) infection has been found to increase the risk of coronary artery disease (CAD) by about $1.5-2$ fold. ${ }^{[1]}$ Conventionally, higher prevalence of traditional risk factors such as smoking, lipid abnormalities and hypertension was implicated. [2] Antiretroviral therapy (ART), particularly protease inhibitors (PI) were also found to have a temporal association because of the positive correlation between lipodystrophy and PI. ${ }^{[3]}$ However, more recent studies indicate that chronic inflammation plays a key role in pathogenesis of CAD in people with HIV. [4] Subclinical atherosclerosis, stable ischemic heart disease, acute 
coronary syndromes have all been described to occur in patients with HIV. ${ }^{[5]}$ However, there has been paucity of studies covering the coronary patterns in such patients.

\section{Objective:}

To assess the pattern of coronary artery disease in patients with HIV by means of coronary angiography.

\section{METHODOLOGY}

The study was conducted in a tertiary care cardiology hospital in central India. The study period was from January to December 2019. Patients admitted for coronary angiography were screened for HIV infection by means of rapid diagnostic kits (Bharat Biotech India ltd, Bengaluru) and if needed by ELISA (Elabsciences, USA). All such patients diagnosed to have HIV were subjected to further routine work up for traditional risk factors and other investigations pertaining to HIV such as CD 4 counts. A complete clinicobiochemical work up was done to arrive at a clinical diagnosis. Patients were classified as having stable ischaemic heart disease/unstable angina /Non ST elevated myocardial infarction/ST elevated myocardial infarction according to the current clinical concepts. Coronary angiography was performed by transradial/femoral route and the cine images were obtained and analysed (GE
Innova IGS 520). The lesions were classified as per the American heart association guidelines for angiographic lesion assessment. ${ }^{[6]}$

\section{RESULTS}

A total of 37 patients presenting to the Cardiology OPD and undergoing coronary angiography were diagnosed to have HIV infection. The mean age was 47 years (19-66) and 27 were males. The baseline characteristics are depicted in table 1.

Table 1: Baseline characteristics in study subjects (HTN Hypertension, DM-Diabetes mellitus, CKD-Chronic kidney disease, SIHD - Stable ischaemic heart disease, UA-Unstable angina, NSTEMI-Non ST elevated myocardial infarction, STEMI-ST elevated myocardial infarction, AWMI-Anterior wall myocardial infarction, IWMI-Inferior wall myocardial infarction, RVMI-Right ventricular myocardial infarction, PWMI-Posterior wall myocardial infarction)

\begin{tabular}{|c|c|c|}
\hline \multirow{3}{*}{$\begin{array}{c}\text { Demographic } \\
\text { characteristics }\end{array}$} & Age & $19-66$ years(47 mean) \\
\cline { 2 - 3 } & Sex & $\begin{array}{c}27-\text { Males(73\%) } \\
10-\text { Females }(27 \%)\end{array}$ \\
\hline Risk factors & HTN & $13(35 \%)$ \\
\cline { 2 - 3 } & DM & $4(11 \%)$ \\
\cline { 2 - 3 } & Dyslipidemia & $17(48 \%)$ \\
\cline { 2 - 3 } & Tobacco & $9(27 \%)$ \\
\cline { 2 - 3 } & CKD & $2(6 \%)$ \\
\cline { 2 - 3 } & CD4 & $67-366(188$ mean) \\
\cline { 2 - 3 } diagnosis & Ounts(cells/cumm) & $31(83 \%)$ \\
\cline { 2 - 3 } & On ART & $8(23 \%)$ \\
\cline { 2 - 3 } & SIHD & $9(24 \%)$ \\
\cline { 2 - 3 } & UA & $4(11 \%)$ \\
\cline { 2 - 3 } & NTSTEMI & $9(24 \%)$ \\
\cline { 2 - 3 } & STEMI - AWMI & $7(19 \%)$ \\
\cline { 2 - 3 } & STEMI - RVMI & 0 \\
\cline { 2 - 3 } & STEMI - PWMI & 0 \\
\hline
\end{tabular}

Note: Decimal points have been rounded off to the nearest integer value

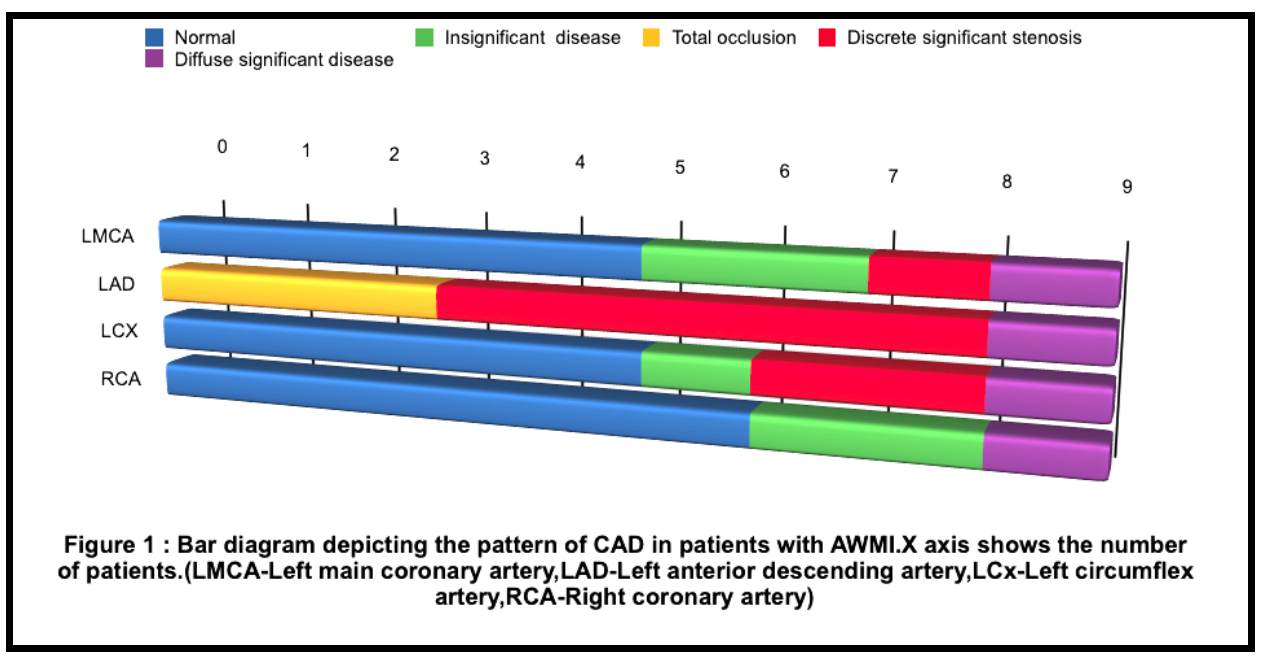

Hypertension was present in $35 \%$ of patients and dyslipidemia (50\%) was the most common traditional risk factor. Non traditional risk factors included low CD 4 counts. They ranged from 67 to 366 with a 
mean of 188cells/cumm. Most of these patients were already on ART (83\%).

Majority of study subjects had myocardial infarction as the first manifestation of HIV related CAD. Of them, AWMI was more common than IWMI. This was followed by NSTEACS (9UA, 4- NSTEMI) and SIHD (8 patients).

\section{Coronary artery pathoanatomy:}

Anterior wall myocardial infarction (Fig 1): When we analysed the pattern of coronary artery anatomy in patients with AWMI, LMCA was found to be normal in majority of patients. Two patients had mild non obstructive plaques, 1 patient had distal $70 \%$ stenosis and 1 patient had diffuse disease. Left anterior descending artery was found to be significantly diseased in all patients; 3 had total occlusions 5 had significant discrete stenosis and one patient had diffusely diseased LAD. Left circumflex was normal in majority of patients, 1 had diffusely ectatic LCx and 2 patients had discrete significant stenosis. Right coronary artery was diffusely stenosed in 1 patient, 2 patients had ectatic and rest had normal RCA.

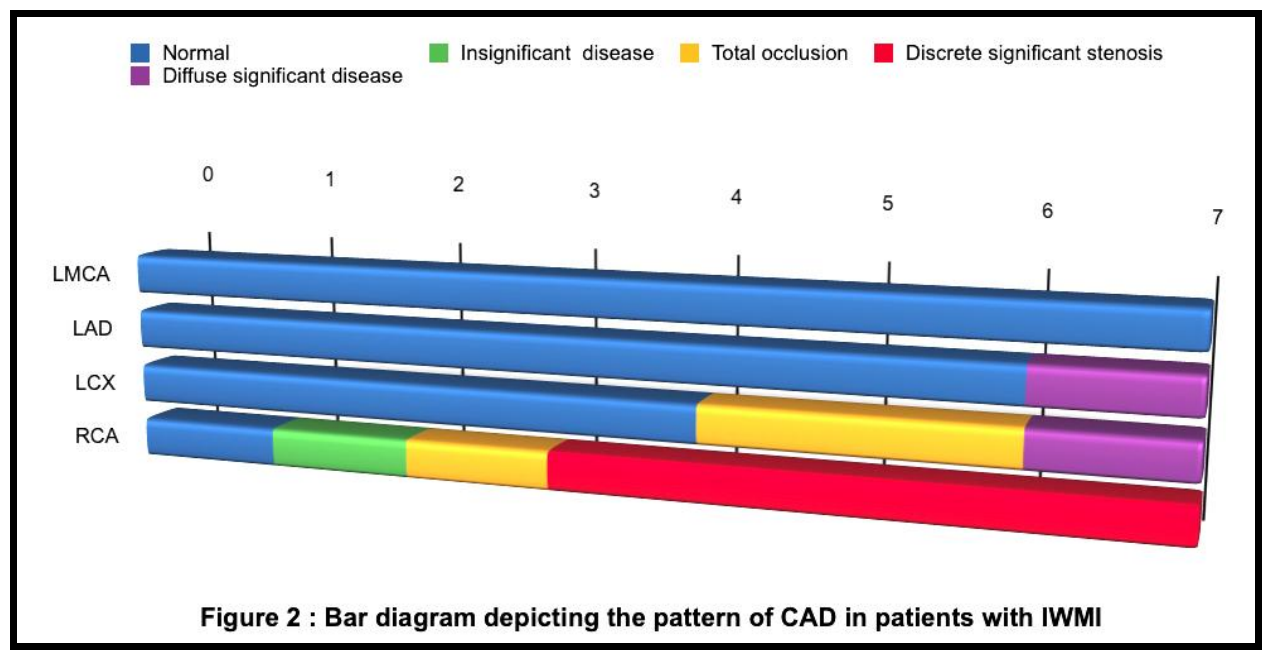

Inferior wall myocardial infarction (Fig 2): All 7 patients with IWMI had normal LMCA, LAD being diffusely diseased in 1 patient. Left circumflex was totally occluded in 2 patients and 1 patient had diffuse significant disease. Right coronary artery had discrete significant stenosis in 4 patients. Total occlusion and ectasia were noted in 1 patient each.

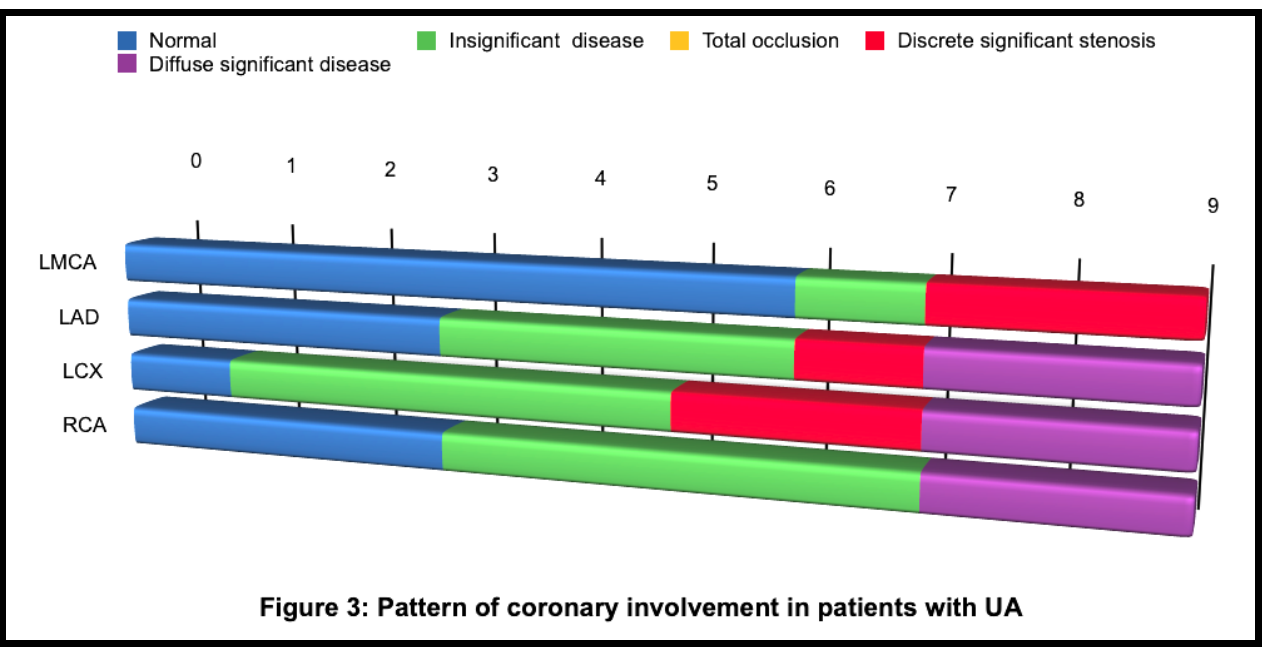

Unstable angina (Fig 3): Left main was normal in most patients, with 1 patient having minor plaque and 2 patients had discrete stenosis. Left anterior descending 
artery had diffuse disease in 5 patients with 2 having significant diffuse disease. Left circumflex was having insignificant disease in 4 patients, discrete stenosis in 2 patients and significant diffuse disease in 2 patients.

Non ST elevated myocardial infarction (Fig 4): LMCA showed discrete insignificant stenosis in 1 patient and the rest had normal LMCA.LAD showed significant discrete stenosis in 2 patients, LCx showed discrete significant stenosis in 3 patients whereas RCA showed discrete stenosis in 3 patients.

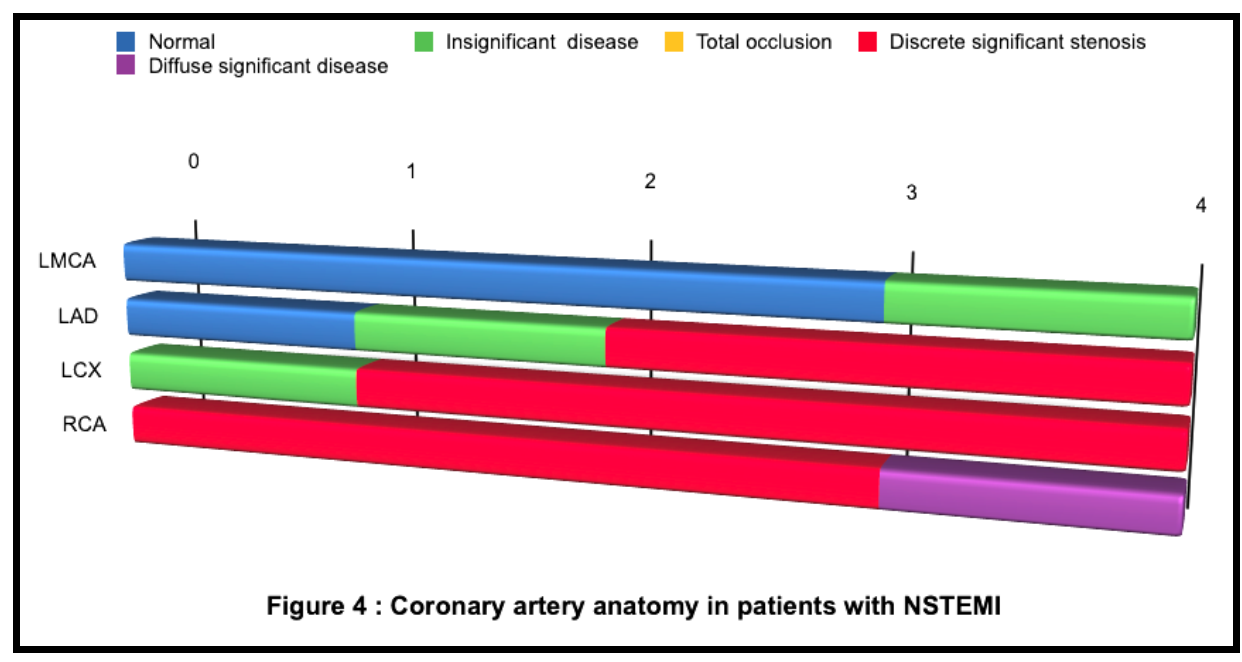

Stable ischaemic heart disease (Fig 5): LMCA was normal in 4 patients. 4 patients had diffusely diseased LMCA, with 3 of them having significant disease. LAD had diffuse in significant stenosis in 4 patients, 1 patient had significant discrete stenosis and 3 patients had significant diffuse disease. LCx and RCA had diffuse disease in majority of patients.

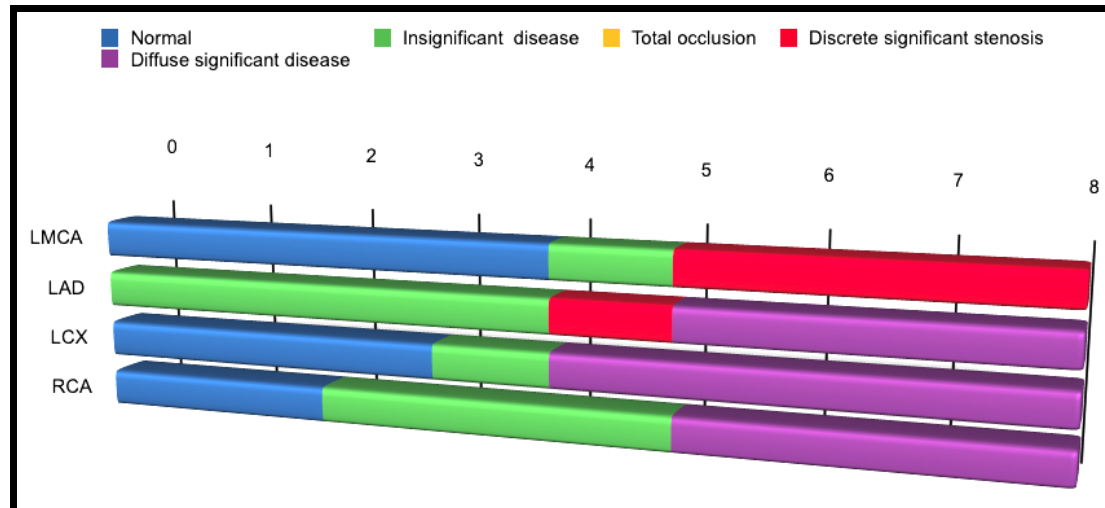

Figure 5 : Coronary profile in patients with stable ischaemic heart disease.

\section{DISCUSSION}

Human immunodeficiency virus infection currently has around 36 million cases globally ${ }^{[7]}$ and leads to a variety of Cardiovascular manifestations in about a third of patients. ${ }^{[8]}$ The proportion of deaths attributable to cardiovascular disease in people living with HIV has doubled between 1999 and 2013, from 2.0\% to 4.6\%. ${ }^{[9]}$ Coronary artery disease ranging from subclinical atherosclerosis to a vulnerable coronary plaque rupture causing acute thrombosis and acute coronary syndrome have been documented with increased frequency. ${ }^{[10]}$ 
Pathogenesis of CAD in HIV involves a complex interplay between traditional and non traditional risk factors (Fig 6). In our study, the youngest study subject was 19 years old and had presented with IWMI. His CAG revealed significant triple vessel disease with $90 \%$ discrete stenosis in LCx and diffusely diseased LAD and RCA. The mean age in our study was 47 years which correlated well with the existing literature. ${ }^{[11]}$

Hypertension as a risk factor has been well documented. In general, HIV patients tend to be more hypertensive as compared to age matched controls. Hypertension, prehypertension lead on to comparatively more incidence of myocardial infarction (MI) in individuals having HIV. ${ }^{[12]}$ Possible etiology has been linked to the endothelial dysfunction and fibrocalcific changes in the blood vessels because of direct effect of HIV infection. ${ }^{[13]}$ In our study, about a third patients were found to be hypertensive.

Diabetes mellitus and dyslipidemia have also been reported to have an increased incidence in patients with HIV. The plausible mechanisms include insulin resistance [14] and HIV/ART induced lipodystrophy. ${ }^{[15]}$ However, only $10 \%$ of our patients had DM whereas dyslipidemia was noted in about a half of patients.

Tobacco chewing/smoking have been increasingly been reported in HIV patients. ${ }^{[16] ~ I n ~ o u r ~ s t u d y, ~ a b o u t ~ a ~ f i f t h ~}$ patients had history of tobacco consumption which is far greater than that seen in age matched controls. ${ }^{[17]}$

Chronic kidney disease was noted in only $2 \%$ patients. Most of our patients were already on ART at the time of presentation and had their CD4 counts ranging from 67 to 366 cells/cumm with a mean of 188 . Although the initial studies depicted a direct link between PI based ART and the incidence of CAD in HIV, the results of the Strategies for Management of Antiretroviral Therapy (SMART) study challenged that concept. [18] More than 5000 HIV participants on ART and with CD4 counts >
350 cells/cumm were randomised to receive either continued ART or interrupted/delayed treatment until CD4 dropped below 250 cells/cumm, which was when ART restarted. Interrupted or deferred ART had a $70 \%$ increased hazard of CVD compared to those who continued treatment. Siedner ${ }^{[19]}$ and other non-randomized studies were also able to demonstrate increased risk of preclinical or clinical CVD among HIV patients with lower nadir CD4 counts. ${ }^{[20]}$ In addition, recent large cohort studies have also demonstrated increased CVD risk among HIV patients with low CD4 counts. [21] These studies suggest that the early initiation of ART (with current guidelines recommending to start ART regardless of immune status) and sustained ART would help reduce CVD risk.

\section{Clinical presentations and their angiographic correlates of CAD in HIV}

The most common clinical presentation in our study was AWMI and UA. Anterior wall myocardial infarction occurred in 9 patients (Age 25-58years). The most commonly diseased artery was LAD. Of these 9 patients, 5 patients had discrete significant stenosis (3 had atherosclerotic $80-90 \%$ stenosis and 2 young females had spontaneous coronary artery dissections. Total occlusions were noted in 3 patients and 1 patient had diffusely diseased LAD. Amongst UA patients, most common coronary presentation was a pan coronary diffuse disease. Acute inferior wall MI was the next common presentation ( 7 patients). Right coronary artery discrete stenosis followed by total occlusions of RCA and LCx were the most commonly observed lesions. Amongst patients with NSTEMI, discrete coronary stenosis affecting RCA and LCx was the most commonly observed lesion. Whereas in patients with stable ischaemic heart disease, LM involvement was quite common (Fig 5) with diffuse disease affecting all other coronaries as the most commonly observed lesion. 
The pathophysiology behind STEMI as the most common presentation possibly is the concept of preferential macrophage differentiation. In chronic HIV infection, there appears to be a differentiation shift into M1 macrophages, which increase cholesterol accumulation and cause disruption of the fibrous cap (compared with M2 macrophages, which contribute to plaque stability). Factors catalysing this preferential M1 differentiation in HIV include soluble CD14 and soluble CD163 proteins, both of which are elevated in HIV. [22] Moreover, macrophages contribute to platelet aggregation, which can lead to coronary occlusion in ACS through the release of tissue factor from foam cells. ${ }^{[23]}$ Thus, this increased activity of macrophages accentuates the formation of arterial plaques, increases their instability, and potentially amplifies their deleterious effects upon rupture in HIV patients. Total occlusions observed in our patients could be plausibly explained by the heightened macrophage activity (M1 subtype).

In our study, the overall coronary picture was dominated by a predominant normal luminogram (Fig 7). A normal luminogram in a patient of HIV however doesnt rule out a preclinical coronary disease. There is growing evidence that HIV patients, whether on ART or not, develop subclinical atherosclerosis. ${ }^{[24]}$ HIV patients in MACS (Multicenter AIDS Cohort Study) were significantly more likely have noncalcified arterial plaque even after adjustment for traditional ASCVD risk factors. ${ }^{[25]}$ There were also greater odds of having significant stenosis of a coronary artery and in this study, low CD4 counts were independently associated with greater likelihood of stenosis.

Diffuse insignificant diseases mainly inclusive of coronary ectasias were not seen infrequently in our study (14 patients) (Fig 8). In some studies, the incidence of ectasias (because of positive remodelling) and coronary artery calcification as detected by non contrast coronary CT angiography have been found to be increased. ${ }^{[26,27]}$ Both of these atherosclerotic features predispose to plaque rupture and may represent a phenotype of elevated risk associated with HIV. The beneficial effect of Statin therapy on reduction of noncalcified plaque volume and high-risk plaque features is being evaluated on a larger scale in the ongoing REPRIEVE study. ${ }^{28]}$

In some studies, the coronary morphology resembling that of transplant vasculopathy (Fig 9) with a characteristic appearance of extensive, concentric fibromuscular hyperplasia of the intima extending through both proximal and distal portions of the epicardial coronary arteries and smaller intramyocardial arteries have been documented. [29] This histologic resemblance further emphasises the potential role of hyperstimulated immune responses and macrophage differentiation shifts as a pathophysiologic basis of HIV associated CAD.

In our study, LAD was the artery which was most frequently affected followed by RCA and the LCx. Left main was least involved. Possible explanations for LAD involvement point to variations in coronary flow dynamics however cannot be highlighted with a statistical/clinical significance.

\section{Strengths and limitations of the study:}

This is one of those rare studies which highlight the angiographic pattern of coronaries in patients with HIV. However, this study has been limited by lack of further in depth analysis of coronary plaque histology pertaining to patients with HIV. Intravascular ultrasound, optical coherence tomography and if feasible histochemical analysis would shed light on the underlying pathophysiological process with the help of which potential therapeutic targets can be further researched upon.

\section{Acknowledgement: None}

\section{Conflict of Interest: None}

\section{Source of Funding: None}


Ethical Approval: Approved

\section{REFERENCES}

1. Grunfeld C, Delaney JA, Wanke C, Currier JS, Scherzer R, Biggs ML, Tien PC, Shlipak MG, Sidney S, Polak JF, O'Leary D, Bacchetti P, Kronmal RA. Preclinical atherosclerosis due to HIV infection: carotid intima-medial thickness measurements from the FRAM study. AIDS. 2009 Sep 10;23(14):1841-9. doi: 10.1097/QAD.0b013e32832d3b85. PMID: 19455012; PMCID: PMC3156613.

2. Friis-Moller N, Weber R, Reiss $\mathrm{P}$, et al. Cardiovascular disease risk factors in HIV patients- association with antiretroviral therapy. results from the DAD study. AIDS (London, England). 2003;17(8):1179-93

3. Bavinger $\mathrm{C}$, Bendavid $\mathrm{E}$, Niehaus $\mathrm{K}$, et al. Risk of cardiovascular disease from antiretroviral therapy for HIV: a systematic review. PloS One. 2013;8(3):e59551

4. Kingsley LA, Cuervo-Rojas J, Munoz A, et al. Subclinical coronary atherosclerosis, HIV infection and antiretroviral therapy: Multicenter AIDS Cohort Study. AIDS (London, England). 2008;22(13):1589-99

5. Currier JS, Taylor A, Boyd F, et al. Coronary heart disease in HIV-infected individuals. J Acquir Immune Defic Syndr. 2003;33(4):506-512

6. Ryan TJ, Faxon DP, Gunnar RM, Kennedy JW, King SB III, Loop FD,Peterson KL, Reeves TJ, Williams DO, Winters WL Jr, et al. Guidelines for percutaneous transluminal coronary angioplasty. A report of the American College of Cardiology/American Heart Association Task Force on Assessment of Diagnostic and Therapeutic Cardiovascular Procedures (Subcommittee on Percutaneous Transluminal Coronary Angioplasty). Circulation 1988;78:486-502

7. Global Health Observatory data on HIV/AIDS int/gho/hiv/en/]

8. Prendergast BD. HIV and cardiovascular medicine. Heart. 2003;89(7):793-800. doi:10.1136/heart.89.7.793

9. Feinstein MJ, Bahiru E, Achenbach C, Longenecker CT, Hsue P, So-Armah K, Freiberg MS, Lloyd-Jones DM. Patterns of cardiovascular mortality for HIV-infected adults in the United States: 1999 to 2013. Am J Cardiol. 2016; 117:214-220
10. Grunfeld C, Delaney JA, Wanke C, Currier JS, Scherzer R, Biggs ML, Tien PC, Shlipak MG, Sidney S, Polak JF, O'Leary D, Bacchetti P, Kronmal RA. Preclinical atherosclerosis due to HIV infection: carotid intima-medial thickness measurements from the FRAM study. AIDS. 2009 Sep 10;23(14):1841-9. 10.1097/QAD.0b013e32832d3b85. PMID: 19455012; PMCID: PMC3156613.

11. Alvaro et al,HIV Infection and Incidence of Cardiovascular Diseases: An Analysis of a Large Healthcare Database;2 Jul 2019https://doi.org/10.1161/JAHA.119.012 241Journal of the American Heart Association. 2019;8:e12241

12. Armah KA, Chang CC, Baker JV, et al. Prehypertension, hypertension, and the risk of acute myocardial infarction in HIVinfected and -uninfected veterans. Clin Infect Dis. 2014;58(1): 121-9.

13. Chi D, Henry J, Kelley J, Thorpe R, Smith JK, Krishnaswamy G. The effects of HIV infection on endothelial function. Endothelium. 2000;7(4):223-42. doi: 10.3109/10623320009072210. PMID: 11201521.

14. Murata H, Hruz PW, Mueckler M. The mechanism of insulin resistance caused by HIV protease inhibitor therapy.J Biol Chem. 2000; 275:20251-20254. doi: 10.1074/jbc.C000228200

15. Grinspoon S, Carr A. Cardiovascular risk and body-fat abnormalities in HIV-infected adults. N Engl J Med. 2005 Jan 6;352(1):4862. doi: 10.1056/NEJMra041811. PMID: 15635112.

16. Petrosillo N, Cicalini S. Smoking and HIV: time for a change? BMC Med. 2013 Jan 22;11:16. doi: 10.1186/1741-7015-11-16. PMID: 23339539; PMCID: PMC3598450.

17. Freiberg MS, Chang CC, Kuller LH, et al. HIV infection and the risk of acute myocardial infarction. JAMA Intern Med. 2013;173(8):614-622.

doi:10.1001/jamainternmed.2013.3728

18. Strategies for Management of Antiretroviral Therapy Study G et al. CD4+ count-guided interruption of antiretroviral treatment. New England J Med. 2006;355(22):2283-96.

19. Siedner MJ. START or SMART? Timing of antiretroviral therapy initiation and cardiovascular risk for people with human immunodeficiency virus infection. Open Forum Infect Dis. 2016;3(1): ofw032 
20. Lang S, Mary-Krause M, Simon A, et al. HIV replication and immune status are independent predictors of the risk of myocardial infarction in $\mathrm{HIV}$-infected individuals. Clin Infect Dis. 2012;55(4): $600-7$

21. Triant VA, Regan S, Lee H, Sax PE, Meigs JB, Grinspoon SK. Association of immunologic and virologic factors with myocardial infarction rates in a US healthcare system. J Acquir Immune Defic Syndr (1999). 2010;55(5):615-9.

22. Hanna DB, Lin J, Post WS, et al. Association of Macrophage Inflammation Biomarkers With Progression of Subclinical Carotid Artery Atherosclerosis in HIVInfected Women and Men. J Infect Dis 2017;215:1352-61

23. Subramanian S, Tawakol A, Burdo TH, et al. Arterial inflammation in patients with HIV. JAMA 2012;308:379-86

24. Thomas GP, Li X, Post WS, et al. Associations between antiretroviral use and subclinical coronary atherosclerosis. AIDS 2016;30:2477-86

25. Post WS, Budoff M, Kingsley L, et al. Associations between HIV infection and subclinical coronary atherosclerosis. Ann Intern Med 2014;160:458-67.

26. Zanni MV, Abbara S, Lo J, Wai B, Hark D, Marmarelis E, Grinspoon SK. Increased coronary atherosclerotic plaque vulnerability by coronary computed tomography angiography in HIV-infected men.AIDS. 2013; 27:1263-1272. doi: 10.1097/QAD.0b013e32835eca9b

27. Fitch KV, Srinivasa S, Abbara S, Burdo TH, Williams KC, Eneh P, Lo J, Grinspoon SK. Noncalcified coronary atherosclerotic plaque and immune activation in HIVinfected women.J Infect Dis. 2013; 208:1737-1746. doi: 10.1093/infdis/jit508

28. Lo J, Lu MT, Ihenachor EJ, Wei J, Looby $\mathrm{SE}$, Fitch $\mathrm{KV}$, Oh J, Zimmerman CO, Hwang J, Abbara S, Plutzky J, Robbins G, Tawakol A, Hoffmann U, Grinspoon SK. Effects of statin therapy on coronary artery plaque volume and high-risk plaque morphology in HIV-infected patients with subclinical atherosclerosis: a randomised, double-blind, placebo-controlled trial. Lancet HIV. 2015; 2:e52-e63. doi: 10.1016/S2352-3018(14)00032-0

29. Ventura HO, Mehra MR, Smart FW, Stapleton DD. Cardiac allograft vasculopathy: current concepts. Am Heart J 1995; 129:791-9

How to cite this article: Rajput AS, Washimkar S, Dalal GS et.al. Cross sectional data on profile of coronary artery disease in human immunodeficiency virus infection patients in a tertiary care hospital of central India. International Journal of Research and Review. 2021; 8(8): 660-667. DOI: https://doi.org/10. 52403/ijrr.20210887 